\title{
Erratum zu: Steuern und Risikobereitschaft in Modellen irreversibler Investitionen
}

\author{
Rainer Niemann • Caren Sureth
}

Online: 1. Dezember 2009

(C) Wirtschaftsuniversität Wien, Austria 2009

\section{Erratum zu: J Betriebswirtsch}

\section{DOI 10.1007/s11301-008-0037-3}

Nach Veröffentlichung des Beitrages baten die Autoren um die Richtigstellung folgender Fehler:

\section{Gleichung (14) lautet richtig:}

$$
\begin{aligned}
& Z^{*}=Z_{s}^{*} \\
& \frac{\lambda}{\lambda-1}(i-\alpha) I=\frac{\lambda_{s}}{\lambda_{s}-1} \frac{i_{s}-\alpha}{1-s}(I-s A) \\
& A^{*}=\frac{1}{s}\left(1-(1-s) \frac{\lambda}{\lambda-1} \frac{\lambda_{s}-1}{\lambda_{s}} \frac{i-\alpha}{i_{s}-\alpha}\right) I, \quad 0<s<1 .
\end{aligned}
$$

The online version of the original article can be found under doi: 10.1007/s11301-008-0037-3

R. Niemann

Institut für Unternehmensrechnung und Steuerlehre, Karl-Franzens-Universität Graz, Universitätsstraße 15, $8010 \mathrm{Graz}$, Österreich

E-Mail: niemann@uni-graz.at

C. Sureth $(\varangle)$

Fakultät für Wirtschaftswissenschaften, Lehrstuhl für Betriebswirtschaftslehre,

insb. Betriebswirtschaftliche Steuerlehre, Universität Paderborn, Warburger Str. 100, 33098 Paderborn, Deutschland

E-Mail: csureth@notes.upb.de 


\section{Abbildung 1 hat den folgenden richtigen Verlauf:}

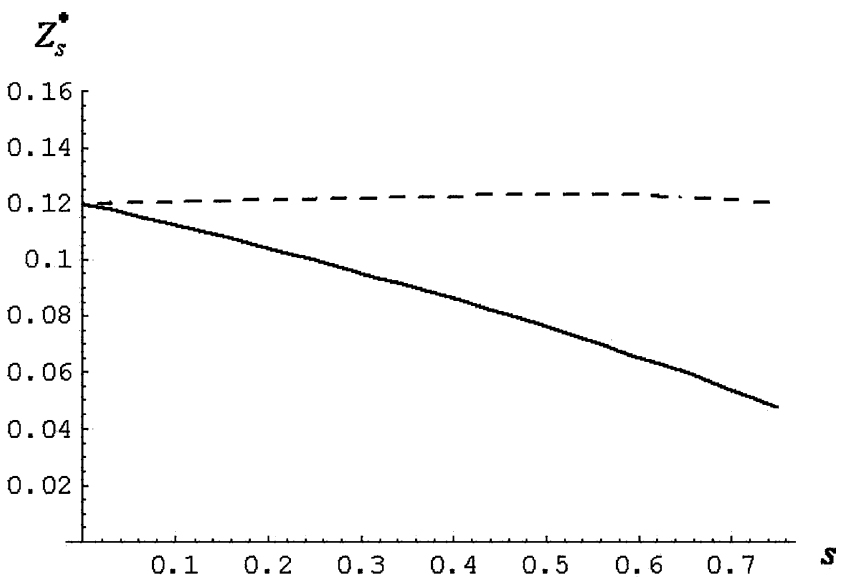

\section{Der Absatz um Abbildung 1 herum (S. 131-132) lautet richtig:}

Aus Abb. 1 geht hervor, dass die Einführung realistischer Steuersätze die kritische Investitionsschwelle im Vergleich zum Vor-Steuer-Fall entweder nur geringfügig erhöhen oder verringern (für $\delta=1 \%$ ) oder aber mehr als halbieren kann (für $\delta=$ $30 \%$ ). Dieser Effekt entspricht dem Steuerparadoxon unter Sicherheit (vgl. Schneider 1969).

Der Verlauf der Funktion für $\delta=30 \%$ erklärt sich einerseits dadurch, dass der Abschreibungsbarwert im Beispiel $A^{*}=\frac{0,3}{0,3+0,08(1-s)}>0$ beträgt und damit den neutralen Abschreibungsbarwert $A^{*}=\frac{3 \sqrt{1-s}-2(1-s)-1}{[4(1-s)-1] s}$ für alle Steuersätze zwischen 0 und $100 \%$ deutlich überschreitet. Der niedrige neutrale Abschreibungsbarwert ist maßgeblich darauf zurückzuführen, dass der Optionswert im hier verwendeten Steuersystem nicht abschreibungsfähig bzw. zuschreibungspflichtig ist. Offensichtlich begünstigt dieses Steuersystem die betrachtete Realinvestition. Bereits anhand dieses einfachen Beispiels wird deutlich, dass die steuerliche Behandlung der Warteoption entscheidenden Einfluss auf die Bereitschaft des Investors zur Risikoübernahme aufweist. 